\title{
Comparative study of Speckle Noise Reduction of Ultrasound B-scan Images in Matrix Laboratory Environment
}

\author{
R. Sivakumar \\ Department of Central Instrumentation \\ \& Service Laboratory \\ University of Madras \\ Maraimalai Campus, Guindy, \\ Chennai, Tamil Nadu, India
}

\author{
D. Nedumaran \\ Assistant Professor \\ Department of Central Instrumentation \\ \& Service Laboratory \\ University of Madras \\ Maraimalai Campus, Guindy, \\ Chennai, Tamil Nadu, India
}

\begin{abstract}
In the field of biomedical imaging, the ultrasound (US) B-Scan images are used for tissue characterization. These images are obtained with a simple linear or sector scan US probe, which show a granular appearance called speckle. Speckle is modeled as a signal dependent noise, which tends to reduce the image resolution and contrast, thereby reducing the diagnostic values of the US imaging modality. Over a period, various speckle reduction techniques have been developed by researchers did not represent a comprehensive method that takes all the constraints into consideration. This work addressed the Wiener filtering in wavelet domain with soft thresholding as a comprehensive technique. Also, this paper compares the efficiency of the wavelet-based thresholding (VisuShrink, BayesShrink and SureShrink) technique in despeckling the medical US images with five other classical speckle reduction filters. The performance of these filters are determined by the statistical quantity measures such as Peak Signal-to-Noise Ratio (PSNR) and Root Mean Square Error (RMSE).The results obtained are presented in the form of filtered images, statistical tables and diagrams. Based on the statistical measures and visual quality of the US B-scan images the Wiener filtering with BayesShrink thresholding technique in the waveletdomian performed well over the other filter techniques.
\end{abstract}

\section{General Terms}

Digital Image Processing

\section{Keywords}

Speckle Reduction, Ultrasound B-Scan image, Image denoising, Wavelet Thresholding, PSNR and RMSE

\section{INTRODUCTION}

The biomedical images obtained by ultrasound (US) systems are significantly poorer compared to other medical imaging systems [14]. But, US images are considered to be non -invasive, portable, accurate, practically harmless to the human beings, [11] and relatively low-cost imaging modality. These features make the ultrasound B-Scan imaging be the most common medical diagnostic tool in hospitals around the world. Unfortunately, the quality of US B-Scan images (as defined by image resolution and contrast) is generally limited due to a number of factors, which originate both from physical phenomena underlying the image acquisition and imperfections of the imaging system design [26]. However, the main drawback of the US image is poor quality of images, which are affected by speckle noise. Speckle is a form of multiplicative noise [13] appears as random mottling of the image with bright and dark spots, which degrades the US images making visual observation quite difficult and limiting their diagnostic potential. There are two basic approaches to speckle reduction in US B-Scan images, one is spatial filtering method and the other one is transform-domain based filtering method. The usual way of removing the speckle noise from US image using the spatial filter technique works well only if the underlying signal is smooth. But, the wavelets give a better performance in image denoising irrespective of the smoothness of the US image due to its sparsity and multiresolution properties. The purpose of the present study was to investigate the speckle reduction techniques of five statistical and three wavelet based speckle suppression filters [2][4] with the objective to remove the speckle noise and to preserve the original image details [16][7][20]. Statistical filters like Frost filter [22][24], Kuan filter, Lee Filter and Wiener filters are chosen for this study due to their efficient speckle reduction property. In addition, a combination of Wiener filter with three soft thresholding techniques have been attempted in the waveletdomain, which exhibits better results than the standard filters [30][2]. Presently, most of the research work in this area aims at developing wavelet thresholding and threshold selection [6] (Hard threshold or Soft threshold) techniques for effective despeckling of US images, since wavelet provides suitable basis functions for separating noisy signal from image signal. Many wavelet based thresholding techniques like VisuShrink, BayesShrink and SureShrink [24] have proved better efficiency in image denoising. To achieve good performance in despeckling, the threshold value must be estimated properly. We described here an efficient thresholding technique by analyzing the statistical parameters of the wavelet coefficients for denoising. The proposed wavelet transform based denoising algorithm consists of the following procedures (i) Calculation the wavelet transform of the noisy signal (ii) Modification of the noisy wavelet coefficients according to a rule (soft thresholding selection) and (iii) Computation of the inverse transform using the modified coefficients [6].

\section{SPECKLE NOISE IN ULTRASOUND IMAGES}

Today, US medical imaging is a common method for diagnosis over the other imaging modalities like Positron emission tomography (PET), Magnetic Resonance imaging (MRI) and Computed tomography (CT) due to its low cost and availability. In monochromatic radiations like US and laser, the radiations scattered from a surface with a roughness of the order of a wavelength produce interference patterns called speckle. To 
understand the speckle noise properties and its despeckling techniques biomedical researchers have been developing mathematical models [27][4][8][19][3]. In this study, general model for speckle noise given by A. K. Jain [14] has been adopted as such and is given in equation 1 .

$$
S(x, y)=f(x, y) \eta_{m}(x, y)+\eta_{a}(x, y)
$$

where $f(x, y)$ is the noise free image to be recovered, $S(x, y)$ is the noisy image, $\eta_{m}(x, y)$ and $\eta_{a}(x, y)$ are multiplicative and additive noises respectively. For any speckle, the contrast ratio $(\xi)$ is defined as

$$
\xi=\frac{\text { Standard deviation of I }}{\text { Mean value of I }}
$$

where I is the intensity field.

\section{SPECKLE REDUCTION USING WAVELET THRESHOLDING}

The recent developments in wavelet transform have firmly recognized its stand as a dominant denoising tool for speckle reduction in medical US images. The following steps perform the wavelet decomposition of the US medical image: In the first stage of the decomposition, spilt the US image into 4 subbands, namely the HH, HL, LH (high pass) and LL (Low pass) subbands [18] [12]. The HH subband gives the diagonal information [6] of the US image; the HL subband gives the horizontal features while the LH subband represents the vertical structures of the US image [2]. The LL subband is the low-resolution residual consisting of low frequency components [28] [21][12] [17] [22] and this subband [5][9][1][10][26] is further divided at the higher levels of decomposition [5][9][25]. The different methods of wavelet denoising investigated so far differ only in the selection of the threshold [23][24][29]. The basic assumption in wavelet de-noising technique is to remove small coefficients, which occurs most likely from noise signals. The steps involved in the Wavelet thresholding method are summarized as under.

- Computation of the wavelet transform from the original image to get the wavelet coefficients of the image using the Discrete Wavelet Transform (DWT). Noise level at each wavelet scale is estimated separately.

- Thresholding the wavelet coefficients (Threshold may be universal or subband adaptive), which defines a threshold of zero to the first wavelet coefficients while the other wavelet coefficients are shrunk accordingly.

- Implementation of the Wiener filter for the LL subband to remove the small noise coefficients.

- Computation of the Inverse Discrete Wavelet Transform (IDWT) to reconstruct the denoised image and to estimate the metrics of the denoised image.

For the threshold selection, soft thresholding [6] has been chosen as the suitable method for noise removal algorithm [18], since it is found to yield visually more pleasing images. On the other hand, hard thresholding is found to introduce artifacts in the recovered images. In this study, three thresholding techniques such as VisuShrink, SureShrink and BayesShrink [24] have been attempted for soft thresholding of the wavelet coefficients and to investigate their performance in denoising the speckle in US Bscan images.

\section{SPECKLE REDUCTION ALGORITHM}

This section describes the method for computing the various parameters used to calculate the threshold value $\left(T_{n}\right)$ [6] [28], which is adaptive to different subband characteristics [22] [9] [1] [24]. The general equation for threshold is given by the equation 3 .

$$
T_{n}=\frac{\gamma \sigma^{2}}{\sigma_{x}}
$$

where $\gamma$ is the scale factor, $\sigma_{\mathrm{x}}$ is the standard deviation of the [12] subband and $\sigma^{2}$ is the variance of the subband.The scale parameter $\gamma$ is calculated for each scale using the following formula.

$$
\gamma=\sqrt{\log \left(\frac{L_{i}}{D_{t}}\right)}
$$

where $L_{i}$ is the length of the subband at the $i^{\text {th }}$ scale, $D_{t}$ is the total number of decompositions. Noise variance $\sigma^{2}$ is estimated using the equation 5 .

$$
\sigma^{2}=\left[\frac{\operatorname{median}\left(\left|Y_{i j}\right|\right)}{0.6745}\right]^{2} Y_{i j} \in \text { Subband HH }
$$

where 0.6745 is the experimental value.

The combination thresholding method applied to the LH, HL, HH subbands and Wiener filtering method applied to the LL subband in the wavelet domain results in a simple and computationally more efficient algorithm for despeckling [18][15]. The complete wavelet algorithm for denoising the speckle can be summarized as follows:

1. Decompose the ultrasound image into subbands.

2. Estimate the noise variance $\left(\sigma^{2}\right)$ in the noisy image using equation (5).

3. For each level, calculate the scale parameter $\gamma$ using the equation (4).

4. For each subband (Except the low pass residual)

(i) Compute the standard deviation and threshold $T_{n}$ using equation (3)

(ii) Apply soft thresholding to the subbands including $\mathrm{LH}_{\mathrm{i}}$, $\mathrm{HL}_{\mathrm{i}}, \mathrm{HH}_{\mathrm{i}}$.

(iii) Apply Wiener filter to the $\mathrm{LL}_{\mathrm{i}}$ subband for removal of noise coefficients.

5. Reconstruct the image from the denoised subbands.

\section{EXPERIMENTAL STUDY AND DISCUSSION}

The US medical image speckle reduction algorithm has been implemented in the MATLAB environment. Various US B-scan images with speckle noise have been obtained from Sri Ramachandra Medical College Hospital, Chennai. The algorithm was tested with three wavelet thresholding and Wiener filtering method in wavelet domain. To estimate the performance of the wavelet filters, five standard filters such as Frost, Lee, Kuan, Wiener and Median have been implemented in this study. The algorithm has been tested for different noise variance values, but the results of despeckling filter of variance values $\sigma^{2}=0.02,0.04$, and 0.06 are summarized here due to its visible and distinct despeckling results. To estimate the performance of despeckling, various statistical values (performance metrics) have been calculated from the denoised US B-Scan images and are found in the literatures [27][30][2][12][16]. In this study, quantitative performance measures such as Root Mean Square Error (RMSE) and Peak Signal-to-Noise Ratio (PSNR) [23][9][24] of the 
ultrasound B-Scan images [25] have been used to estimate the filter performance. The RMSE is calculated using the equation 6 .

$$
R M S E=\sqrt{\frac{\sum(q(i, j)-Q(i, j))^{2}}{M N}}
$$

where $\mathrm{MN}$ is the number of pixel of the US B-Scan image, and $q(i, j)$, and $Q(i, j)$ represent the original and denoised images respectively. The PSNR is the ratio between [23] the maximum possible power of a signal and the power of corrupting noise that affects the fidelity of its representation. The PSNR value is calculated using the equation 7.

$$
\begin{array}{r}
P S N R=20 \log _{10} \frac{255}{\sqrt{\frac{\sum(q(i, j)-Q(i, j))^{2}}{M N}}} \\
\text { or } \\
P S N R=20 \log _{10} \frac{255}{R M S E}
\end{array}
$$

The despeckling filter algorithm developed in the MATRIX LABORATORY (MATLAB) environment, have been tested in more than 200 US B-scan images of different organs. The test results of three patients of the US B-Scan images namely Echogenic-liqor, Bicornuate-pregnancy and Umbilical-vein-varix are given in figs 1-3. Also the performance metrics calculated from the denoised image of the different filters are summarized in Tables 1-3 for comparison. From the performance metrics given in Tables 1-3, and visual inspection of the denoised images, wavelet thresholding with Wiener filter techniques performed well over the other standard speckle filters, since the low RMSE value and high PSNR value results in better denoising. Wiener and Median filter alone also gave comparable results with the Wavelet filters. But Median filter represents the additive noise characteristics better as compared to the multiplicative noises like speckle. Wiener is the better approach as per as standard filters are concerned, since it has performance metrics comparable with the outperformed Wavelet filters. But Wavelet thresholding with Wiener filter has exceptional performance than Wiener in terms of metrics and visual inspection. Of the three thresholding scheme employed in wavelet filter, BayesShrink performed better than the other two thresholding schemes.

\section{CONCLUSION}

In this paper we proposed a simple and efficient algorithm for speckle noise reduction of the US B-Scan image, which combines the Wiener filtering and thresholding methods in the wavelet transform domain. Experiments are conducted on three different ultrasound images corrupted by speckle noise to assess the performance of wavelet thresholding techniques viz., VisuShrink, BayesShrink and SureShrink. Also, a comparative study has been made between the Wiener filter with Wavelet thresholding and other standard filters like Frost, Lee, Kuan, Median and Wiener filters. The out come of this study reveals that Wiener filter with BayesShrink thresholding scheme outperforms all other filters interms of RMSE, PSNR and visual quality.

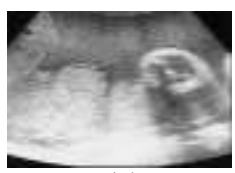

(a)

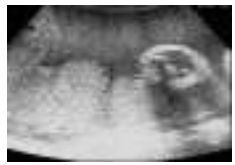

(d)

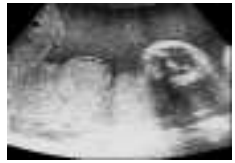

(g)

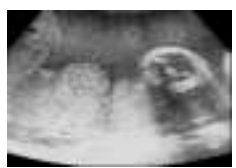

(b)

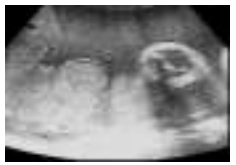

(e)

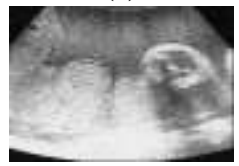

(h)

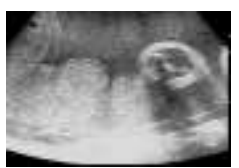

(c)

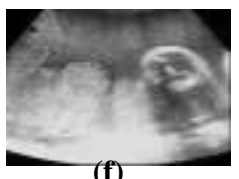

(f)

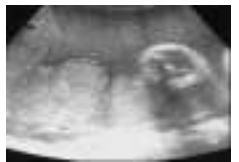

(i)
Fig 1 US B-scan image of the echogenic-liqor image (a) Original image, (b) VisuShrink, (c) Kuan filter, (d) Median filter, (e) Frost filter, (f) BayesShrink, (g) Lee filter, (h) Weiner filter and (i) SureShrink

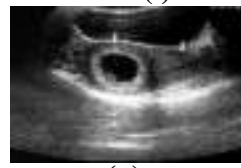

(a)

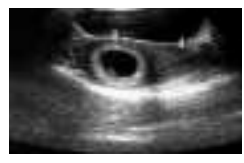

(d)

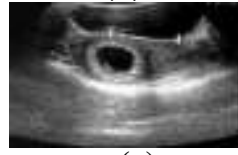

(g)

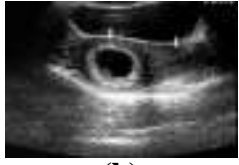

(b)

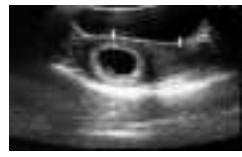

(e)

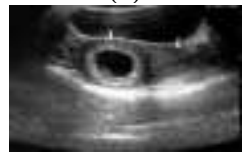

(h)

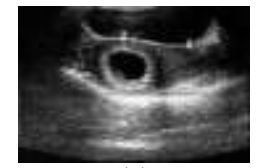

(c)

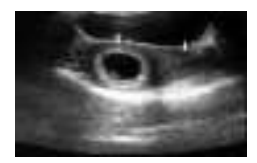

(f)

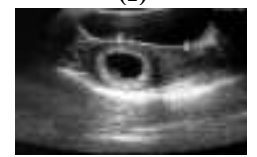

(i)
Fig 2 US B-scan image of the bicornuate-pregnancy image (a) Original image, (b) VisuShrink, (c) Kuan filter, (d) Median filter, (e) Frost filter, (f) BayesShrink, (g) Lee filter, (h) Weiner filter and (i) SureShrink

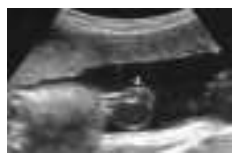

(a)

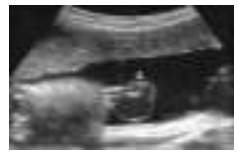

(d)

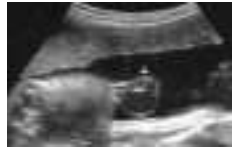

(g)

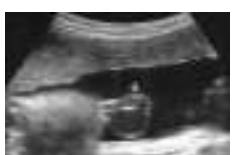

(b)

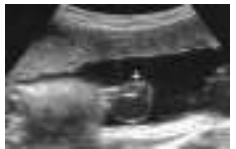

(e)

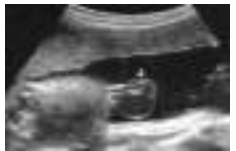

(h)

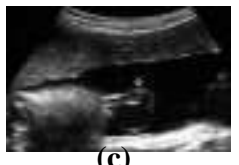

(c)

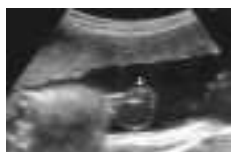

(f)

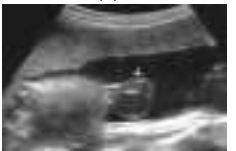

(i)
Fig 3 US B-scan image of the Umbilical-vein-varix image (a) Original image, (b) VisuShrink, (c) Kuan filter, (d) Median filter, (e) Frost filter, (f) BayesShrink, (g) Lee filter, (h) Weiner filter and (i) SureShrink 
Table 1 Comparison of PSNR \& RMSE values of different denoising filters for Echogenic-liqor Ultrasound images corrupted by speckle noise

\begin{tabular}{|c|c|c|c|c|c|c|}
\hline \multirow{2}{*}{ Filters } & \multicolumn{2}{|c|}{$\sigma^{2}=0.02$} & \multicolumn{2}{c|}{$\sigma^{2}=0.04$} & \multicolumn{2}{c|}{$\sigma^{2}=0.06$} \\
\cline { 2 - 7 } & RMSE & PSNR & RMSE & PSNR & RMSE & PSNR \\
\hline Frost & 17.58 & 23.22 & 19.80 & 22.19 & 23.33 & 20.76 \\
\hline Kaun & 16.70 & 23.67 & 19.85 & 22.16 & 22.37 & 21.13 \\
\hline Visu & 5.94 & 32.64 & 7.23 & 30.94 & 9.64 & 28.43 \\
\hline Bayes & 5.61 & 33.14 & 6.49 & 31.87 & 8.12 & 29.93 \\
\hline Wiener & 5.72 & 32.96 & 7.17 & 31.00 & 9.23 & 28.81 \\
\hline Sure & 5.80 & 32.84 & 7.39 & 30.74 & 9.11 & 28.93 \\
\hline Lee & 19.99 & 22.10 & 24.58 & 20.31 & 28.95 & 18.89 \\
\hline Median & 5.86 & 32.76 & 7.75 & 30.33 & 9.79 & 28.30 \\
\hline
\end{tabular}

Table 2.Comparison of PSNR \& RMSE values of different denoising filters for bicornuate- Pregnancy ultrasound images corrupted by speckle noise

\begin{tabular}{|c|c|c|c|c|c|c|}
\hline \multirow{2}{*}{ Filters } & \multicolumn{2}{|c|}{$\sigma^{2}=0.02$} & \multicolumn{2}{c|}{$\sigma^{2}=0.04$} & \multicolumn{2}{c|}{$\sigma^{2}=0.06$} \\
\cline { 2 - 7 } & RMSE & PSNR & RMSE & PSNR & RMSE & PSNR \\
\hline Frost & 17.68 & 23.17 & 20.16 & 22.03 & 24.34 & 20.39 \\
\hline Kaun & 17.34 & 23.34 & 19.41 & 22.36 & 23.21 & 20.80 \\
\hline Visu & 6.12 & 32.38 & 7.47 & 30.65 & 9.93 & 28.18 \\
\hline Bayes & 5.68 & 33.03 & 6.62 & 31.69 & 8.39 & 29.64 \\
\hline Wiener & 5.70 & 33.00 & 7.20 & 30.97 & 9.32 & 28.73 \\
\hline Sure & 5.70 & 32.99 & 7.19 & 30.98 & 9.02 & 29.01 \\
\hline Lee & 20.39 & 21.97 & 25.15 & 20.12 & 29.56 & 18.71 \\
\hline Median & 5.72 & 32.96 & 7.45 & 30.67 & 9.42 & 28.64 \\
\hline
\end{tabular}

Table 3.Comparison of PSNR \& RMSE values of different denoising filters for Umbilical-vein- varix ultrasound images corrupted by speckle noise.

\begin{tabular}{|c|c|c|c|c|c|c|}
\hline \multirow{2}{*}{ Filters } & \multicolumn{2}{|c|}{$\sigma^{2}=0.02$} & \multicolumn{2}{c|}{$\sigma^{2}=0.04$} & \multicolumn{2}{c|}{$\sigma^{2}=0.06$} \\
\cline { 2 - 7 } & RMSE & PSNR & RMSE & PSNR & RMSE & PSNR \\
\hline Frost & 14.98 & 24.61 & 17.18 & 23.42 & 22.54 & 21.06 \\
\hline Kaun & 14.60 & 24.83 & 16.13 & 23.96 & 20.09 & 22.06 \\
\hline Visu & 6.10 & 32.41 & 7.14 & 31.04 & 8.71 & 29.32 \\
\hline Bayes & 5.61 & 33.14 & 6.48 & 31.89 & 8.04 & 30.01 \\
\hline Wiener & 5.75 & 32.91 & 6.74 & 31.54 & 8.08 & 29.96 \\
\hline Sure & 5.87 & 32.74 & 7.20 & 30.96 & 8.39 & 29.64 \\
\hline Lee & 20.12 & 22.02 & 22.28 & 21.19 & 26.26 & 19.74 \\
\hline Median & 6.35 & 32.06 & 7.39 & 30.74 & 9.12 & 28.92 \\
\hline
\end{tabular}

\section{ACKNOWLEDGMENTS}

We acknowledge the help extended by Prof. Dr. S. Thanikachalam, Chairman, Cardiac Care Centre, SRMC Hospital, and Chennai for providing various ultrasound B-scan images to conduct this study. Also, we record the financial assistance provided by TamilNadu State Council for Science Technology (TNSCST), Chennai to carry out this work

\section{REFERENCES}

[1] Alin Achim., Anastasios Bezerianos., and Panagiotis Tsakalides. 2001. Novel Bayesian Multiscale Method for Speckle Removal in Medical Ultrasound Images. IEEE Transactions on Medical imaging, Vol. 20, No. 8, pp.772- 783.

[2] Aleksandra Pǐzurica, Wilfried Philips, Ignace Lemahieu, and Marc Acheroy. 2003. A Versatile Wavelet Domain Noise Filtration Technique for Medical Imaging. IEEE Transactions on medical imaging Vol.22, No.3, pp. 323-331.
[3] Antonio Fernandez - Caballero, and Juhan, L. Mateo. 2008. Methodological Approach to Reducing Speckle Noise in Ultrasound images. International Conference on Biomed. Engie. And Informatics, IEEE computer society, 978-0-7695-3118-2/08.

[4] Dainty, J.C. 1971. Detection of images immersed in speckle noise. Optica Acta, Vol. 18, No. 5, pp.327-339.

[5] David L. Donoho, and Iain M.Johnstone.1994. Ideal Spatial adaptation via wavelet shrinkage. Biometrika, Vol. 81, pp.425-455.

[6] David L. Donoho.1995. De-Noising by Soft-Thresholding. IEEE Transactions on information theory, Vol.41, No. 3, pp. 613627.

[7] Evans, A.N., and Nixon, M.S. 1993. Speckle filtering in ultrasound images for feature extraction. IEEE Conf. of Acoustic Sensing and Imaging, 369, pp.44-49.

[8] Goodman, J.W. 1976. Some fundamental properties of speckle. Journal of Optical Society of America, Vol. 66, No. 11, pp. $1145-1150$

[9] Grace Chang, S., Bin Yu., and Vattereli, M. 2000. Adaptive Wavelet Thresholding for Image denoising and Compression. IEEE Transactions on Image Processing, Vol. 9, pp.1532- 1546.

[10] Grace Chang, S., Bin Yu., and Vattereli, M. 2000. Spatially Adaptive Wavelet Thresholding with Context Modeling for Image denoising. IEEE Transaction on Image Processing, Vol. 9, pp.1522-1530.

[11] Gjenna Stippel, Wilfried Philips, Ignace Lemahieu, Paul Govart. 2002. A New Medical Feature Enhancing speckle Suppression method for Ultrasound images of Neonatal Brain. Proc. of (359) Signal and Image Processing.

[12] Gnanadurai, D., and Sadasivam,V. 2003. An Efficient Adaptive Thresholding Technique for Wavelet Based image Denoising. International Journal of Signal Processing Vol.2, No. 2, pp.114-119.

[13] Gonzalez, Rafael C.,Woods, Richard E., Eddins, Steven L. 2004. Digital Image Processing Using MATLAB ${ }^{\circledR}$. Pearson Education, Inc.

[14] Jain, A.K. 1989. Fundamental of Digital Image Processing. Englewood Cliffs, NJ: Prentice-Hall.

[15] Khaled Z., Abd -Elmoniem, Abou-Bakr M., Youssef, and Yasser M ., Kadah. 2002. Real -Time Speckle Reduction and Coherence Enhancement in Ultrasound Imaging via Nonlinear Anisotropic Diffusion. IEEE Transactions on Biomed. Engine, Vol. 49, No. 9, pp. 997-1014.

[16] Loupas, T., Mcdicken, W.N., and Allan, P.L. 1989. An Adaptive Weighted Median Filter for Speckle Suppression in Medical Ultrasonic Images. IEEE Transactions on circuits and systems, Vol.36, No.1, pp.129-135. 
[17] Motwani, M.C., Gadiya, M.C., Motwani, R.C., Frederick., and Harris, C. Jr. 2004. Survey of Image Denoising Techniques. Proceedings of GSPx, Santa Clara, CA.

[18] Mario Mastriani. 2006. New Wavelet- Based Superresolution Algorithm for Speckle Reduction in SAR Images. Inter. Journal of Comp. Sci., Vol.1, No.4, pp.291-298.

[19] Robert F. Wagner, Stephen W. Smith, John M. Sandrik, and Hector Lopez.1983. Statistics of Speckle in Ultrasound B-Scans. IEEE Transactions on Sonics and Ultrasonic, Vol. 30, No. 3, pp.156-163

[20] Richard N. Czerwinski, Douglas L. Jones., and William D.O' Brien, Jr.1995. Ultrasound speckle reduction by directional median filtering. IEEE, 0 -8186-7310-9/95, pp.358-361.

[21] Su Cheol Kang, and Seung Hong. 2001. Experimental and Theoretical Analysis of Wavelet based denoising filter for Echocardiographic images. MEDINFO, pp-906-909.

[22] Sudha, S., Suresh, G.R., Sukanesh, R. 2007. Wavelet Based Image Denoising using Adaptive Thresholding. IEEE Computer Society, Intern. Conf. on Computational Intelligence and Multimedia applications, pp. 296 - 300, 2007.

[23] Sivakumar, R., and Nedumaran, D. 2009. Performance Study of Wavelet denoising techniques in Ultrasound images. Journal of Instrument Society of India, Vol.39, No.3, pp.194-196.

[24] Sudha, S., Suresh, G.R., and Sukanesh, R. 2009. Speckle noise reduction in ultrasound images using context - based adaptive wavelet Thresholding. IETE Journal of Research, Vol.55, Issue 3.
[25] Thangavel, K. Manavalan, R., and Laurence Aroquiaraj, I.. 2009. Removal of Speckle Noise from Ultrasound Medical Image based on Special Filters: Comparative Study. ICGST-GVIP Journal, ISSN 1687-398X, Vol. 9, Issue (III), pp.25-32.

[26] Wavelet and image processing Toolbox for Matlab commercial package include in Matrix Laboratory (http://www.mathworks.com).

[27] Xiaohui Hao, Shangkai Gao, and Xiaorong Gao. 1999. A Novel Multiscale Nonlinear Thresholding Method for Ultrasonic Speckle Suppressing. IEEE Transactions on Medical imaging, Vol.18, No.9, pp.787-794.

[28] Yong Sun Kim, and Jong Beom Ra. 2005. Improvement of Ultrasound Image Based on Wavelet Transform: Speckle Reduction and Edge Enhancement. Medical imaging Proc. of SPIE, Vol.5747, pp.1085-1092.

[29] Yuan Chen, and Amar Raheja. 2005. Wavelet Lifting for Speckle Noise Reduction in Ultrasound Images. IEEE Proc. of the Engine. In Medicine and Biology, $27^{\mathrm{th}}$ Annual Conference, pp.3129-3132.

[30] Zhang, L.C.,Wong, E.M.C., Koh,L.M., and Ng, L.S. 2004. An Adaptive Filter for Speckle Reduction in Medical Ultrasound Image Processing. 8th Inter. Conf. on Control, Automation, Robotics and Vision Kunming, China, pp.654-658. 